\title{
High Resolution Surface Morphology Measurements using EBSD Cross-Correlation Techniques and AFM
}

\author{
M.D. Vaudin, G. Stan, Y.B. Gerbig and R.F. Cook
}

Ceramics Division, National Institute of Standards and Technology, Gaithersburg, MD

Electron backscatter diffraction (EBSD) cross-correlation techniques [1] were recently applied to the strain fields surrounding a wedge indentation in silicon, and the computed stresses compared with those measured using confocal Raman microscopy. Very good agreement between these stress measurements was obtained [2], thus validating the methods as able to measure stresses with nano-scale resolution. This work has been extended into the area of morphology measurement, using the antisymmetric component of the deformation gradient tensor measured by EBSD, rather than the symmetric component used in strain measurement. Using both EBSD in the scanning electron microscope (SEM) and atomic force microscopy (AFM), the deformation fields surrounding $20 \mu \mathrm{m}$ long $350 \mathrm{mN}$ wedge indentations in (001) Si were measured.

In the EBSD method, the indentation is aligned with the $y$-axis of the SEM stage and a scan is performed in the $x$-direction across the center of the indentation, collecting high-quality EBSD patterns from the sample surface. A pattern obtained from a strain-free part of the sample is chosen as the reference and the other patterns are cross-correlated against this reference; selected regions of interest (ROIs) in each pattern are compared. The technique determines the complete deformation gradient tensor, which can be separated into strain and rigid body rotations tensors. Integrating the rotation tensor element about the $y$-axis $(\partial z / \partial x)$ along the $x$-direction allows the height of the sample above the reference position to be determined, thus producing an EBSD height profile across the indentation. The step size for the scan was $0.2 \mu \mathrm{m}$ which was sufficiently small that the integration could be achieved by a simple summation without introducing a detectable error. The samples were cleaned for AFM observation using a replica technique. For AFM imaging, using intermittent contact mode, the scan direction was aligned perpendicular to the indentation impression (as for EBSD) and $x$-scan lines over the central 10 $\mu \mathrm{m}$ of the indentation were averaged to produce an AFM height profile for comparison with the EBSD height profile.

Fig. 1 is an AFM image of a $350 \mathrm{mN}$ wedge indentation, with a 3-D inset. The elevated region around the indentation extends approximately $10 \mu \mathrm{m}$ on either side of the impression, which is over $150 \mathrm{~nm}$ deep. Fig. 2 shows a height profile comparison for the elevated region. The very good agreement between the EBSD and AFM height profiles is clear. Outside the elevated region, the AFM image indicates a small amount of roughness on the sample surface of the order of $2 \mathrm{~nm}$ which is apparent in Fig. 2. The EBSD plot does not indicate this roughness as the roughness was not accompanied by a change in crystallographic orientation.

References

[1] A.F. Wilkinson, G. Meaden, and D.J. Dingley, Ultramicroscopy 106, 307 (2006)

[2] M.D. Vaudin, Y.B. Gerbig, S.J. Stranick and R.F. Cook, Appl. Phys. Letts. 93, 193116 (2008) 
Fig 1:

_AFM images of $350 \mathrm{mN}$ wedge indentation in $\mathrm{Si}(001)$.

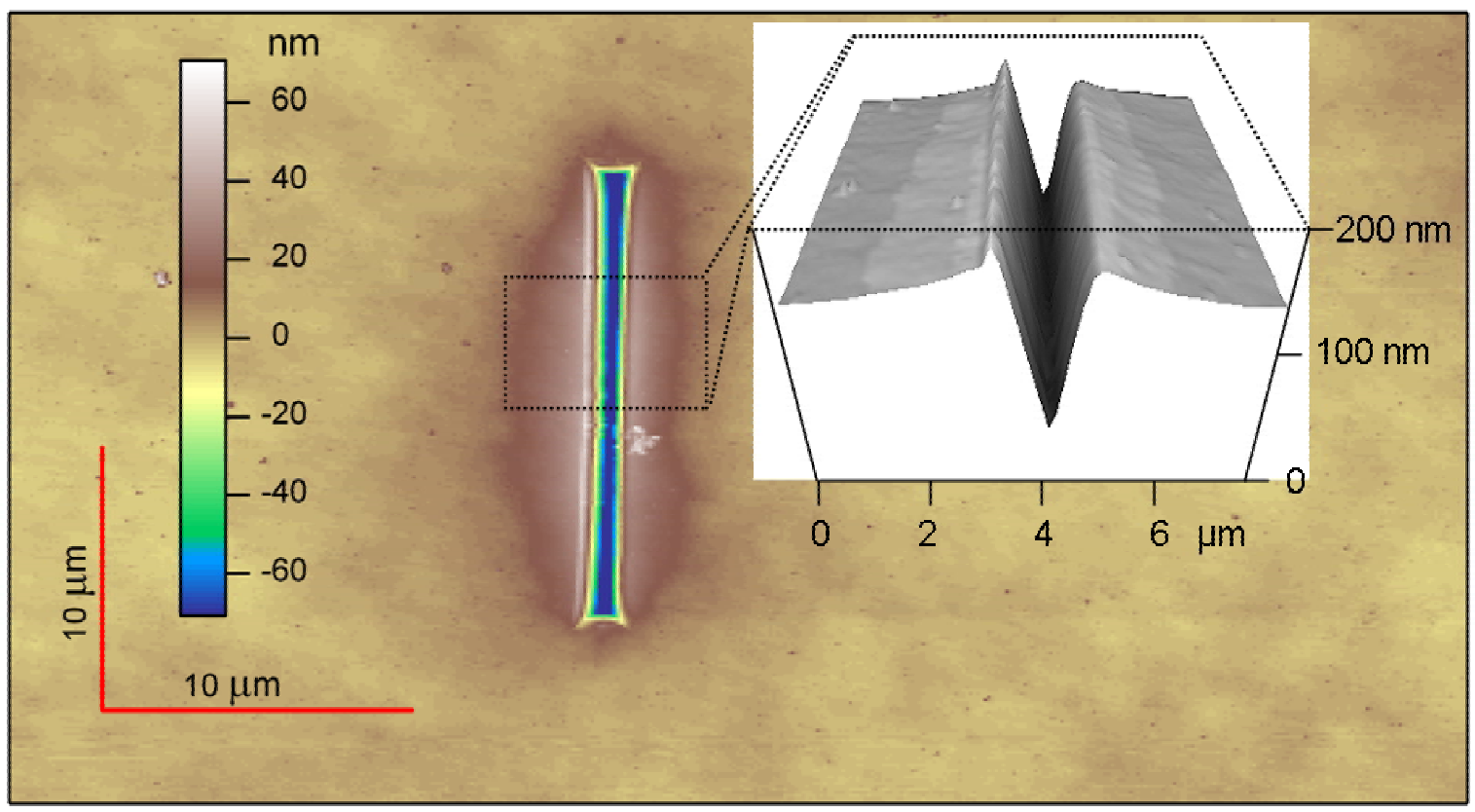

Fig 2:

Comparison of height profiles across wedge indentation in Si determined by EBSD and AFM.

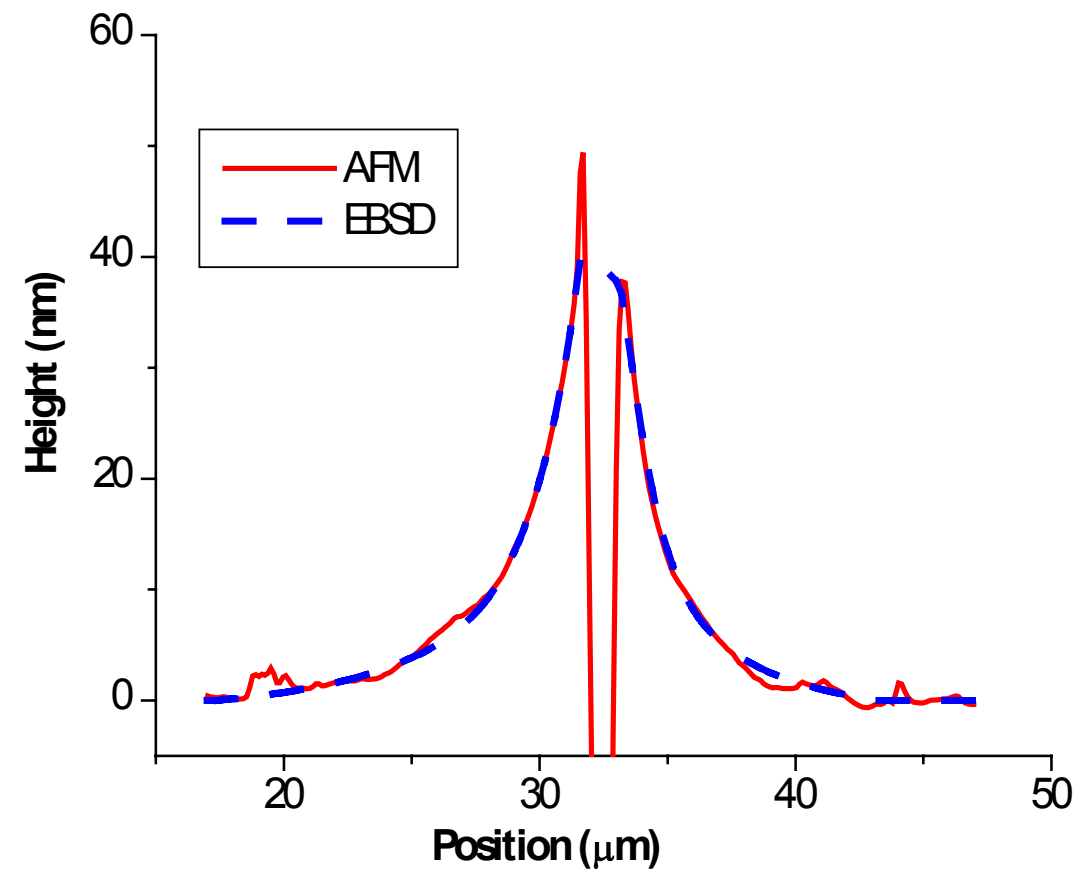

